\title{
New Reports of Wild Mushroom Diversity from Foothill region of Uttarakhand
}

\author{
Vasundhra Sharma ${ }^{1 *} \mathcal{E}$ A. K. Jaitly ${ }^{2}$
}

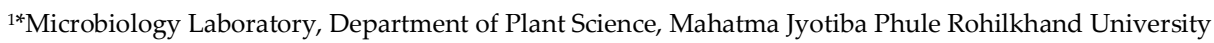
Bareilly-243006; researchdhara@gmail.com; +917906877143

2 Microbiology Laboratory, Department of Plant Science, Mahatma Jyotiba Phule Rohilkhand University Bareilly-243006; jaitly1958@gmail.com; +917906295951

\begin{abstract}
A The present investigation was undertaken in foothill regions of Uttarakhand from July-2016 up to December-2018. A total of thirty four different sites ranging from the roadside areas, grasslands to forests were studied and Mushroom fruiting bodies were collected. A total of One Hundred sixty six fruiting counts were obtained and 68 mushroom genera belonging to 15 orders and 43 families were identified. During collection visits mushroom were apparent from organic debris of diversified habitats ranging from humid soil; grassland; leaf litter; living tree trunk; dead wood log of forest zone. Maximum fruiting bodies (75\%) were obtained between July to September and minimum i.e. $6 \%$ between November - February. Among the collected mushroom Stereum rugosum, Crepidotus variabilis, Laccaria laccata, Schizophyllum commune, Ganoderma applantum, Cantharellus cibarius were more prevalent. Out of all collected mushroom sample the frequency of Mushroom belonging to order Agaricales was 45.18\% followed by Polyporales i.e., $27.7 \%$. The collected mushroom were cultured on PDA medium and their mycelial forms were preserved for further studies.
\end{abstract}

Keywords: Mushroom, organic-debris, fruiting bodies, diversity, frequency.

\section{Introduction}

The Macro-fungi having fleshy, sub-fleshy, leathery, and umbrella like fructifications, bearing their spore producing surface either on lamellae (gills) or lining the tubes, opening out by means of pores designated as Mushroom [1]. These fruiting bodies falls under the category of gilled mushroom, bracket mushroom, puffballs, coral mushroom, jelly mushroom, stinkhorns, earthstars, birds nest mushroom on the testimony of their sporocarps [2]. From the taxonomic point of view, mainly Basidiomycetes are known as mushroom but also some species of Ascomycetes have been known to produce mushroom like fruiting bodies [3]. Mushroom are inseparable parts of ecosystem being soil replenisher due to their strong property of degrading cellulose and other organic polymers. The fact marks presence or absence of mushroom are useful trafficator to assess the damage or the maturity of an ecosystem [4]. Mushroom in the $21^{\text {st }}$ century has been explored as crucial component for food safety and security. They have rich nutritional value with high content of proteins, vitamins, minerals, fibers, trace elements, limited calories and cholesterol [5]. Besides, they also contain good amount of secondary metabolites and were reported to possessed antioxidant, anticancer, anti-mutagenic, antimicrobial and antiradical properties [6]. The rate of consumption of fleshy fungi in many countries has increased in recent years and hence it becomes imperative to explore the treasure of wild mushrooms. The day by day increasing population and its developmental activities are detrimental to ecological diversity. India is rich in diversity of its fauna and flora consequently may possess virtuous heterogeneity of Mushroom. The first scientific study on Indian mushroom was handled by Linnaeus in $18^{\text {th }}$ century with the identification and description of Podaxis pistillaris [7]. Several mycologists have reported 
ethno-mycological usage and additional lists appeared in between $18^{\text {th }}$ to $21^{\text {st }}$ century culminating with this natural resource wealth of India. The studies on mushrooms are mainly aimed at describing only common cultivating varieties and validating its status but, there are relatively a few studies that have been undertaken on wild mushroom mainly due to their short life span of their fruiting bodies. Ecological value, diversity and economic importance of mushroom are three key aspects in the world of biology [8]. However, indigenous knowledge about edible and medicinal mushrooms has not been given significant attention in Foothill region of Uttarakhand. The cities of Uttarakhand have an upscale repository of the unexplored macro-fungal wealth due to its varied climatic and topographic conditions. Therefore, present study has been undertaken to explore the biodiversity of mushroom from Foothill region of Uttarakhand especially of Kumaon region.

\section{Materials and Methods}

Regular collection visits were conducted in different months during July-2016 up to December-2018 from Bareilly to Uttarakhand. Prior and during the collection from selected sites a standard protocol had been followed [9]. The study area was located between $28^{\circ} 43^{\prime}-31^{\circ} 27^{\prime} \mathrm{N}$ latitudes and $77^{\circ} 34^{\prime}-81^{\circ} 02^{\prime}$ E longitudes, $64 \%$ of which is covered with alpine rain forest. The Foothills of Uttarakhand in the south is delimited by Bareilly region of Uttar Pradesh. It has a subtropical climate and the weather is cool to pleasant. The temperature ranges between $32^{\circ} \mathrm{C}-4^{\circ} \mathrm{C}$. The study area has been divided into four sub areas i.e., 1.Nainital; 2.Udham Singh Nagar; 3.Haridwar;4.Garhwal in conjunction with foothill bordering geographical region i.e., 5.Bareilly (fig1.) A total of thirty four sites were selected from above mentioned sub areas for the collection of fruiting bodies in different environmental conditions. The full bloomed and complete fruiting bodies of fresh samples were photographed with the aid of Nikon D3400 camera and collected from their natural habitats. All of the physicochemical parameters of collection sites were recorded with the help of a multimeter of Cutezy and a digital thermometer. The entire collected Mushroom were brought to Microbiology Laboratory under aseptic conditions in cooling thermocol containers for Microscopic examination under microscope (cell image centre Llyod) and further evaluation. Identification had been undertaken as per mentioned procedure at the sites www.rogersmushrooms.com, www.mushroomexpert.com and comparing the external morphology, pictures and microscopic slides preparations with standard manual and field guide $[10,11,12]$ Isolation of pure culture of fruiting body had been obtained on PDA media and pure forms were stored at $4^{\circ} \mathrm{C}$ for further biochemical and physicochemical analysis. The Frequency of the collected mushroom was calculated by the following formula:

$$
\text { Frequency of mushroom species }=\frac{\text { Number of sites in which the species occured }}{\text { Total no of sites }} \times 100
$$

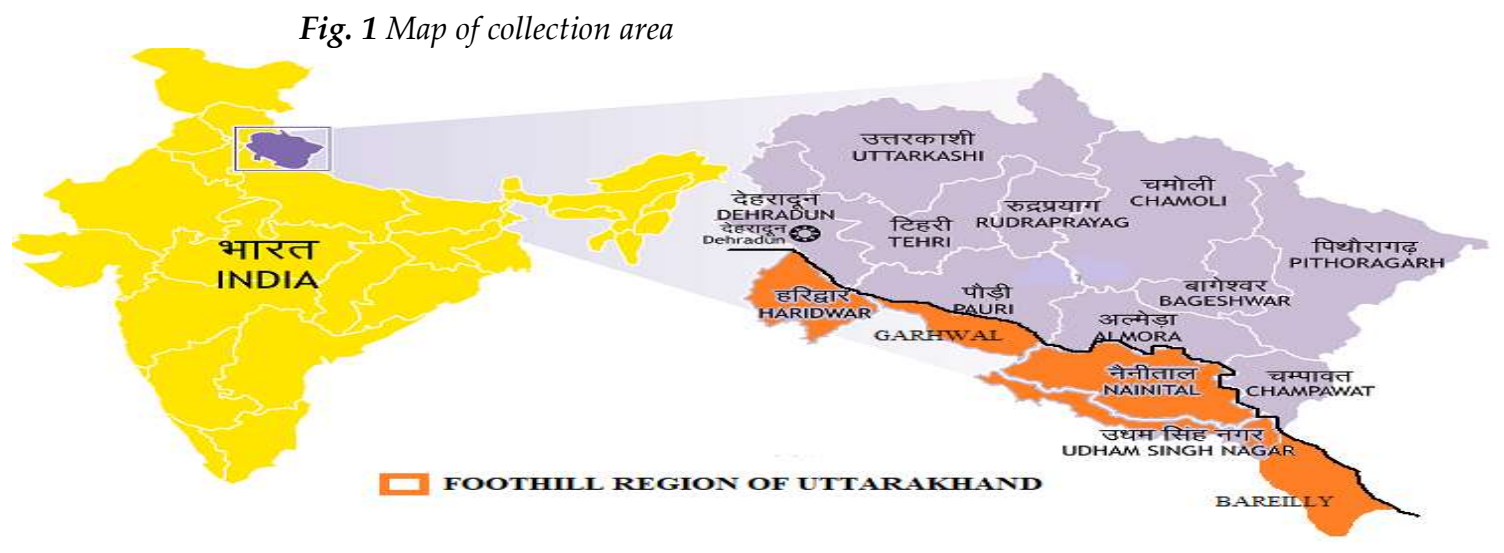




\section{Results and Discussion}

This research paper represents the new report made on wild mushroom from Foothill region of Uttarakhand, India. A total of 166 Mushroom samples were collected and identified. The collected mushroom were found to grew naturally in different habitats like grasslands, living tree trunk, under soil, dead wood log, leaf litter etc. in different environmental conditions. Most of the collected mushrooms were found to prevail from July to November due to the favorable temperature and humidity of that time span for fruiting bodies to bloom (table 1). Generally good amount of Rainfall occurs during the month of August, thus decreasing the emergence of fruiting bodies followed by the highest collection percentage in September $(30 \%)$ with average day temperatures of $18^{\circ} \mathrm{C}$ dropping at night to $4^{\circ} \mathrm{C}$. The occurrence and collection per cent of mushroom has been recorded predominantly where $\mathrm{pH}$ was ranging from 7.1-7.5 (table 1.). Agaricales and Polyporales emerged out to be the dominant mushroom orders (fig. 2.) in the studied region with their per cent frequencies 45.18 and 27.7 alternatively. Out of total 166 identified samples; 102 different species of 15 orders and 43 families were identified. Among identified mushroom Crepidotus variabilis, Stereum rugosum, Ganoderma applantum, Schizophyllum commune were more prevalent followed by gilled mushroom i.e. Laccaria laccta, Cantherallus cibarius and Chlorophyllum molybdites whereas jelly fungi were rarely found viz. Dacrymyces palmatus, Auricularia mesentrica (table2.).

Table 1: Average Physicochemical Parameters of Sites

\begin{tabular}{|c|c|c|c|c|c|}
\hline Site & Code & Temperature $\left({ }^{\circ} \mathrm{C}\right)$ & pH & Light (lux) & Moisture \\
\hline Lalkuan & $S 1$ & 29 & 7.2 & 1200 & 6 \\
\hline Haldwani & $S 2$ & 33 & 6.2 & 1000 & 3 \\
\hline Kathgodam & $S 3$ & 25 & 7.5 & 800 & 4 \\
\hline Gola Beraj & $S 4$ & 26 & 8 & 600 & 8 \\
\hline Dolmar & 55 & 23 & 7.8 & 300 & 10 \\
\hline Jeolikot & 56 & 22 & 7.2 & 200 & 9 \\
\hline Amdanda & $S 7$ & 21 & 7.2 & 200 & 8 \\
\hline Garjiya & 58 & 31 & 5.9 & 500 & 3 \\
\hline Kaladhungi & 59 & 25 & 7.1 & 400 & 5 \\
\hline Ramnagar & $S 10$ & 22 & 7.3 & 500 & 3 \\
\hline Pantnagar & $S 11$ & 31 & 7.5 & 800 & 4 \\
\hline Haldi & $\$ 12$ & 29 & 7.3 & 700 & 3 \\
\hline Pattarcahata & 513 & 29 & 7.2 & 600 & 3 \\
\hline Mundeli & $\$ 14$ & 27 & 7.6 & 900 & 2 \\
\hline Pari & $\$ 15$ & 26 & 7.5 & 700 & 2 \\
\hline Khatima city & 516 & 30 & 7.8 & 1000 & 3 \\
\hline Nanakmatta & $\$ 17$ & 31 & 7.8 & 1200 & 3 \\
\hline Sitarganj & 518 & 32 & 7.9 & 1000 & 4 \\
\hline Jaspur & $S 19$ & 31 & 7.6 & 900 & 5 \\
\hline Gadarpur & $S 2 O$ & 31 & 7.5 & 800 & 3 \\
\hline Rudrapur & $S 21$ & 30 & 7.6 & 600 & 3 \\
\hline Chidiyapur & $\mathrm{S} 22$ & 25 & 6.9 & 500 & 8 \\
\hline Laksar & $\mathrm{S} 23$ & 30 & 7.8 & 700 & 3 \\
\hline Jwalapur & $\mathbf{S} 24$ & 29 & 7.7 & 600 & 6 \\
\hline Raiwala & $\mathrm{S} 25$ & 26 & 7.8 & 300 & 4 \\
\hline Chiddarwala & 526 & 25 & 6.9 & 200 & 7 \\
\hline Asafpur & $\mathbf{S 2 7}$ & 31 & 7.6 & 800 & 3 \\
\hline Landsowne & $\mathbf{S} 28$ & 23 & 7.5 & 400 & 7 \\
\hline Dogadda & 529 & 22 & 7.7 & 300 & 5 \\
\hline Kotdwar & $\mathrm{S} 30$ & 26 & 7.1 & 600 & 7 \\
\hline University & $\mathrm{S} 31$ & 30 & 6.8 & 500 & 7 \\
\hline Kargaina & $\mathrm{S} 32$ & 29 & 7.2 & 900 & 2 \\
\hline Subhash Nagar & $\mathrm{S} 33$ & 32 & 7.3 & 1000 & 3 \\
\hline C.B. Ganj & $\mathrm{S} 34$ & 28 & 7.5 & 700 & 8 \\
\hline
\end{tabular}


Table 2. Mushroom Diversity of Foothill region of Uttarakhand

\begin{tabular}{|c|c|c|c|c|c|}
\hline SITE & SAMPLE & IDENTIFIED SPECIES & OCCURRENCE & ORDER & $\%$ FREQUENCY \\
\hline \multirow{10}{*}{ S1 } & M1 & Mycena polygramma & Grassland & Agaricales & 8.82 \\
\hline & $\mathrm{M} 2$ & Pycnoporus sanguineus & Leaf litter & Polyporales & 8.82 \\
\hline & M3 & Coprinus plicatilis & Leaf litter & Agaricales & 5.88 \\
\hline & M4 & Hygrocybe pratensis & Base of Stem & Agaricales & 5.88 \\
\hline & M5 & Dacrymyces palmatus & Base of Stem & Dacrymycetales & 2.94 \\
\hline & M6 & Chlorophyllum molybdites & Leaf litter & Agaricales & 11.76 \\
\hline & M7 & Trametes versicolor & Dead woodlog & Polyporales & 8.82 \\
\hline & M8 & Stereum rugosum & Leaf litter & Rusullales & 14.71 \\
\hline & M9 & Daedalea quercina & Deadwoodlog & Polyporales & 5.88 \\
\hline & M10 & Crepidotus variabilis & Base of Stem & Agaricales & 14.71 \\
\hline \multirow{4}{*}{$\mathrm{S} 2$} & M11 & Pleurocybella porrnigens & Base of Stem & Agaricales & 5.88 \\
\hline & M12 & Laccaria laccata & Base of Stem & Agaricales & 11.76 \\
\hline & M13 & Coltricia perennis & Grassland & Hymenochaetales & 5.88 \\
\hline & M14 & Mycena polygramma & Grassland & Agaricales & 8.82 \\
\hline \multirow{7}{*}{ S3 } & M15 & Volvopleutus gloiocephalus & Base of Stem & Agaricales & 5.88 \\
\hline & M16 & Hygrocybe pratensis & Base of Stem & Agaricales & 5.88 \\
\hline & M17 & Auricularia auricula judae & Dead woodlog & Auriculariales & 8.82 \\
\hline & M18 & Chlorociboria aeruginascens & Leaf litter & Helotiales & 2.94 \\
\hline & M19 & Pleurotus ostreatus & Leaf litter & Agaricales & 5.88 \\
\hline & M20 & Pycnoporus cinnabarinus & Leaf litter & Polyporales & 2.94 \\
\hline & M21 & Marasmius alliaceus & Base of Stem & Agaricales & 5.88 \\
\hline \multirow{5}{*}{ S4 } & M22 & Pycnoporus sanguineus & Dead woodlog & Polyporales & 8.82 \\
\hline & M23 & Coprinopsis atramentaria & Leaf litter & Agaricales & 2.94 \\
\hline & M24 & Coltricia perennis & Grassland & Hymenochaetales & 5.88 \\
\hline & M25 & Volvopleutus gloiocephalus & Base of Stem & Agaricales & 5.88 \\
\hline & M26 & Ganoderma curtissi & Wood log & Polyporales & 2.94 \\
\hline \multirow{5}{*}{ S5 } & M27 & Macrolepiota procera & Grassland & Agaricales & 8.82 \\
\hline & M28 & Lycoperdon pyriforme & Grassland & Agaricales & 5.88 \\
\hline & M29 & Hexagonia spp. & Leaf litter & Polyporales & 2.94 \\
\hline & M30 & Pleurocybella porrnigens & Base of Stem & Agaricales & 5.88 \\
\hline & M31 & Tricholoma sulphurium & Grassland & Agaricales & 5.88 \\
\hline \multirow{6}{*}{ S6 } & M32 & Coprinus domesticus & Leaf litter & Agaricales & 5.88 \\
\hline & M33 & Stereum rugosum & dead woodlog & Rusullales & 14.71 \\
\hline & M34 & Leptoporus adustus & Deadwoodlog & Polyporales & 2.94 \\
\hline & M35 & Coprinus plicatilis & Leaf litter & Agaricales & 5.88 \\
\hline & M36 & Ganoderma adspersum & Wood log & Polyporales & 2.94 \\
\hline & M37 & Climacodon septentrionalis & Base of stem & Polyporales & 2.94 \\
\hline \multirow{10}{*}{ S7 } & M38 & Calvatia craniiformis & Leaf litter & Agaricales & 5.88 \\
\hline & M39 & Laccaria bicolor & Leaf litter & Agaricales & 5.88 \\
\hline & M40 & Cantharellus minor & Grassland & Cantharellales & 5.88 \\
\hline & M41 & Laccaria laccata & Grassland & Agaricales & 11.76 \\
\hline & M42 & Daedalea quercina & Woodlog & Polyporales & 5.88 \\
\hline & M43 & Trametes versicolor & Dead woodlog & Polyporales & 8.82 \\
\hline & M44 & Mycena marginata & Dead woodlog & Agaricales & 8.82 \\
\hline & M45 & Crepidotus variabilis & Base of stem & Agaricales & 14.71 \\
\hline & M46 & Schizophyllum commune & Living tree base & Agaricales & 14.71 \\
\hline & M47 & Mycena polygramma & Base of stem & Agaricales & 8.82 \\
\hline \multirow{4}{*}{ S8 } & M48 & Lycoperdon pyriforme & Grassland & Agaricales & 5.88 \\
\hline & M49 & Chlorophyllum molybdites & Leaf litter & Agaricales & 11.76 \\
\hline & M50 & Crepidotus variabilis & Grassland & Agaricales & 14.71 \\
\hline & M51 & Xylaria polymorpha & Woodlog & Xylariales & 2.94 \\
\hline
\end{tabular}




\begin{tabular}{|c|c|c|c|c|c|}
\hline \multirow{11}{*}{ S9 } & M52 & Meripilus giganteus & Leaf litter & Polyporales & 2.94 \\
\hline & M53 & Cantharellus minor & Base of stem & Cantharellales & 5.88 \\
\hline & M54 & Schizophyllum commune & Saprophtic & Agaricales & 14.71 \\
\hline & M55 & Ganoderma applantum & Base of stem & Polyporales & 14.71 \\
\hline & M56 & Crepidotus variabilis & Leaf litter & Agaricales & 14.71 \\
\hline & $\overline{M 57}$ & Laccaria bicolor & Leaf litter & Agaricales & 5.88 \\
\hline & $\overline{M 58}$ & Pleurotus ostreatus & Woodlog & Agaricales & 5.88 \\
\hline & $\overline{M 59}$ & Trametes gibbosa & Woodlog & Polyporales & 2.94 \\
\hline & M60 & Polyporus alveolaris & Base of stem & Polyporales & 2.94 \\
\hline & M61 & Coprinus comatus & Dead woodlog & Agaricales & 2.94 \\
\hline & $\overline{M 62}$ & Tricholoma sulphurium & Grassland & Agaricales & 5.88 \\
\hline \multirow{6}{*}{ S10 } & M63 & Amanita virosa & Woodlog & Agaricales & 2.94 \\
\hline & M64 & Schizophyllum commune & Tree trunk & Agaricales & 14.71 \\
\hline & M65 & Lentinus tigrinus & Woodlog & Polyporales & 2.94 \\
\hline & M66 & Psathyrella conopilus & Grassland & Agaricales & 2.94 \\
\hline & M67 & Collybia dryophila & Dead wood & Agaricales & 2.94 \\
\hline & $\overline{M 68}$ & Lactarius deliciosus & Living treebase & Russulales & 5.88 \\
\hline \multirow{3}{*}{ S11 } & M69 & Entoloma conferendum & Mycorrhizal & Agaricales & 2.94 \\
\hline & $\overline{M 70}$ & Schizophyllum commune & Mycorrhizal & Agaricales & 14.71 \\
\hline & M71 & Clitocybe gibba & Grassland & Agaricales & 5.88 \\
\hline \multirow{3}{*}{$\mathrm{S} 12$} & M72 & Mycena marginata & Leaf litter & Agaricales & 8.82 \\
\hline & M73 & Hygrocybe calyptiformis & Grassland & Agaricales & 2.94 \\
\hline & $\overline{M 74}$ & Agaricus sylvaticus & Leaf litter & Agaricales & 2.94 \\
\hline \multirow{2}{*}{$\mathrm{S} 13$} & M75 & Cantharellus cibarius & Debris & Cantharellales & 11.76 \\
\hline & M76 & Cliptopilus prunulus & Debris & Agaricales & 2.94 \\
\hline \multirow{2}{*}{ S14 } & M77 & Auricularia auricula judae & Deadwood $\log$ & Auriculariales & 8.82 \\
\hline & M78 & Cantherellus cibarius & Grassland & Cantharellales & 2.94 \\
\hline \multirow{2}{*}{ S15 } & M79 & Condrostereum pupureum & Leaf litter & Agaricales & 2.94 \\
\hline & $\overline{M 80}$ & Sparassis crispa & Woodlog & Polyporales & 8.82 \\
\hline \multirow{5}{*}{ S16 } & M81 & Tyromyces chioneus & Deadwood log & Polyporales & 2.94 \\
\hline & M82 & Auricularia mesenrtica & Living tree trunk & Auriculariales & 5.88 \\
\hline & M83 & Polyporus brumalis & Deadwood log & Polyporales & 2.94 \\
\hline & M84 & Cortinarius spp. & Debris & Agaricales & 2.94 \\
\hline & M85 & Xylaria hypoxylon & Deadwood log & Xylariales & 8.82 \\
\hline \multirow{5}{*}{ S17 } & M86 & Coriolus versicolor & Living Tree trunk & Polyporales & 5.88 \\
\hline & M87 & Cantherallus subalbidus & Leaf litter & Cantharellales & 2.94 \\
\hline & M88 & Daedaleopsis confragosa & Living tree trunk & Polyporales & 5.88 \\
\hline & M89 & Gymnophus dryophilus & Deadwood log & Agaricales & 2.94 \\
\hline & M90 & Trametes hirsuta & Deadwood log & Polyporales & 2.94 \\
\hline \multirow{6}{*}{ S18 } & M91 & Ganoderma applantum & Living tree trunk & Polyporales & 14.71 \\
\hline & M92 & Ganoderma resinaceum & Deadwood log & Polyporales & 8.82 \\
\hline & M93 & Macrolepiota procera & Grassland & Agaricales & 8.82 \\
\hline & M94 & Auricularia auricula judae & Deadwood log & Auriculariales & 8.82 \\
\hline & M95 & Gleophyllum odoratum & Deadwood log & Gleophyllales & 2.94 \\
\hline & M96 & Ganoderma applantum & Living tree trunk & Polyporales & 14.71 \\
\hline \multirow{2}{*}{ S19 } & M97 & Pseudohydnum gelatinosum & Deadwood log & Auriculariales & 2.94 \\
\hline & M98 & Mycena marginata & Debris & Agaricales & 8.82 \\
\hline \multirow{2}{*}{ S20 } & M99 & Disciotis venosa & Deadwood log & Pezizales & 2.94 \\
\hline & M100 & Podoscypha petalodes & Deadwood log & Polyporales & 2.94 \\
\hline $\bar{S} 21$ & M101 & Hymenochaete rubiginosa & Deadwood $\log$ & Hymenochaetales & 2.94 \\
\hline \multirow{6}{*}{ S22 } & M102 & Lenzites betulina & Deadwood log & Polyporales & 2.94 \\
\hline & M103 & Phlebia tremellosa & Livilg tree trunk & Polyporales & 2.94 \\
\hline & M104 & Polyporus umbellatus & Leaflitter & Polyporales & 2.94 \\
\hline & M105 & Stereum rugosum & Deadwood $\log$ & Rusullales & 14.71 \\
\hline & M106 & Xylaria hypoxylon & Leaflitter & Xylariales & 8.82 \\
\hline & M107 & Ganoderma resinaceum & Deadwood log & Polyporales & 8.82 \\
\hline \multirow{2}{*}{ S23 } & M108 & Cymatoderma spp. & Living tree trunk & Agaricales & 2.94 \\
\hline & M109 & Heterobasidium annosum & Deadwood log & Rusullales & 2.94 \\
\hline
\end{tabular}




\begin{tabular}{|c|c|c|c|c|c|}
\hline \multirow{3}{*}{ S24 } & M110 & Crepidotus porringens & Living tree trunk & Agaricales & 5.88 \\
\hline & M111 & Gloeophyllum sepiarium & Deadwood log & Gleophyllales & 5.88 \\
\hline & M112 & Cantharellus cibarius & Leaflitter & Cantharellales & 11.76 \\
\hline \multirow{8}{*}{ S25 } & M113 & Sparassis crispa & Deadwood log & Polyporales & 8.82 \\
\hline & M114 & Clitocybe nuda & Grassland & Agaricales & 2.94 \\
\hline & M115 & Polyporus squamosus & Deadwood log & Polyporales & 2.94 \\
\hline & M116 & Hygrophoris aurantiaca & Grassland & Boletales & 2.94 \\
\hline & M117 & Ramaria stricta & Leaflitter & Dacrymycetales & 2.94 \\
\hline & M118 & Phellinus spp. & Living tree trunk & Hymenochaetales & 2.94 \\
\hline & M119 & Lactarius deliciosus & Living tree trunk & Russulales & 5.88 \\
\hline & M120 & Fomes fomantarius & Woodlog & Polyporales & 2.94 \\
\hline \multirow{10}{*}{ S26 } & M121 & Crepidotus porringens & Living tree trunk & Agaricales & 5.88 \\
\hline & M122 & Clitocybe gibba & Grassland & Agaricales & 5.88 \\
\hline & M123 & Mycena pelianthena & Leaflitter & Agaricales & 2.94 \\
\hline & M124 & Lenzites tigrinus & Leaflitter & \begin{tabular}{|l|} 
Polyporales \\
\end{tabular} & 2.94 \\
\hline & M125 & Thelophora terrstris & Leaflitter & Thelophorales & 2.94 \\
\hline & M126 & Flemmulinas velutipes & Leaflitter & Agaricales & 2.94 \\
\hline & M127 & Lactarius trivialis & Grassland & Rusullales & 2.94 \\
\hline & M128 & Pleurotus sajor caju & Living tree trunk & Agaricales & 2.94 \\
\hline & M129 & Ganoderma applantum & Deadwood log & Polyporales & 14.71 \\
\hline & M130 & Trichaptum abietum & Leaflitter & Polyporales & 2.94 \\
\hline \multirow{2}{*}{ S27 } & M131 & Clitopilus prunulus & Deadwood log & Agaricales & 2.94 \\
\hline & M132 & Pycnoporus sanguineus & Deadwood log & Polyporales & 8.82 \\
\hline \multirow{7}{*}{$\mathrm{S} 28$} & M133 & Daedaleopsis confragosa & Deadwood log & Polyporales & 5.88 \\
\hline & M134 & Gloeophyllum sepiarium & Living tree trunk & Gloeophyllales & 5.88 \\
\hline & M135 & Laetiporus sulphurius & Living tree trunk & Polyporales & 2.94 \\
\hline & M136 & Scleroderma verucosum & Grassland & Boletales & 2.94 \\
\hline & M137 & Bondarzawia berkeleyi & Dead woodlog & Russullales & 2.94 \\
\hline & M138 & Tyromyces stipticus & Dead woodlog & Polyporales & 2.94 \\
\hline & M139 & Schizophyllum commune & Dead woodlog & Agaricales & 14.71 \\
\hline \multirow{3}{*}{ S29 } & M140 & Cantharellus cibarius & Grassland & Cantharellales & 11.76 \\
\hline & M141 & Crelophus cirrhatus & Living tree trunk & Russullales & 2.94 \\
\hline & M142 & Phellodon tomentosus & Grassland & Thelophorales & 2.94 \\
\hline \multirow{4}{*}{$\mathrm{S} 30$} & M143 & Sparassis crispa & Dead woodlog & Polyporales & 8.82 \\
\hline & M144 & Auricularia mesenrtica & Dead woodlog & Auriculariales & 5.88 \\
\hline & M145 & Bovista plumbae & Grassland & Agaricales & 2.94 \\
\hline & M146 & Tremella mesentrica & Dead woodlog & Tremellales & 2.94 \\
\hline \multirow{9}{*}{ S31 } & M147 & Chlorophyllum molybdites & Leaf litter & Agaricales & 11.76 \\
\hline & M148 & Laccaria laccata & Leaf litter & Agaricales & 11.76 \\
\hline & M149 & Irpex lacteus & Deadwood log & Polyporales & 5.88 \\
\hline & M150 & Calvatia utriformes & Grassland & Agaricales & 2.94 \\
\hline & M151 & Crepidotus variabilis & Living tree trunk & Agaricales & 14.71 \\
\hline & M152 & Cantharellus cibarius & Grassland & Cantharellales & 11.76 \\
\hline & M153 & Marasmius sullivanti & Grassland & Agaricales & 2.94 \\
\hline & M154 & Ganoderma resinaceum & Deadwood $\log$ & Polyporales & 8.82 \\
\hline & M155 & Agaricus bisporus & Ground & Agaricales & 2.94 \\
\hline \multirow{4}{*}{ S32 } & M156 & Ganoderma applantum & Living tree trunk & Polyporales & 14.71 \\
\hline & M157 & Chlorophyllum molybdites & Leaf litter & Agaricales & 11.76 \\
\hline & M158 & Laccaria laccata & Grassland & Agaricales & 11.76 \\
\hline & M159 & Stereum rugosum & Deadwood $\log$ & Rusullales & 14.71 \\
\hline \multirow{3}{*}{ S33 } & M160 & Macrolepiota procera & Grassland & Agaricales & 8.82 \\
\hline & M161 & Trametes versicolor & Living Tree trunk & Polyporales & 8.82 \\
\hline & M162 & Xylaria hypoxylon & Deadwood log & Xylariales & 8.82 \\
\hline \multirow{4}{*}{ S34 } & M163 & Coprinus domesticus & Leaf litter & Agaricales & 5.88 \\
\hline & M164 & Calvatia craniiformis & Grassland & Agaricales & 5.88 \\
\hline & M165 & Marasmius alliaceus & Base of Stem & Agaricales & 5.88 \\
\hline & M166 & Stereum rugosum & Deadwood log & Rusullales & 14.71 \\
\hline
\end{tabular}


Fig.2: Frequency of Mushroom Orders from studied region

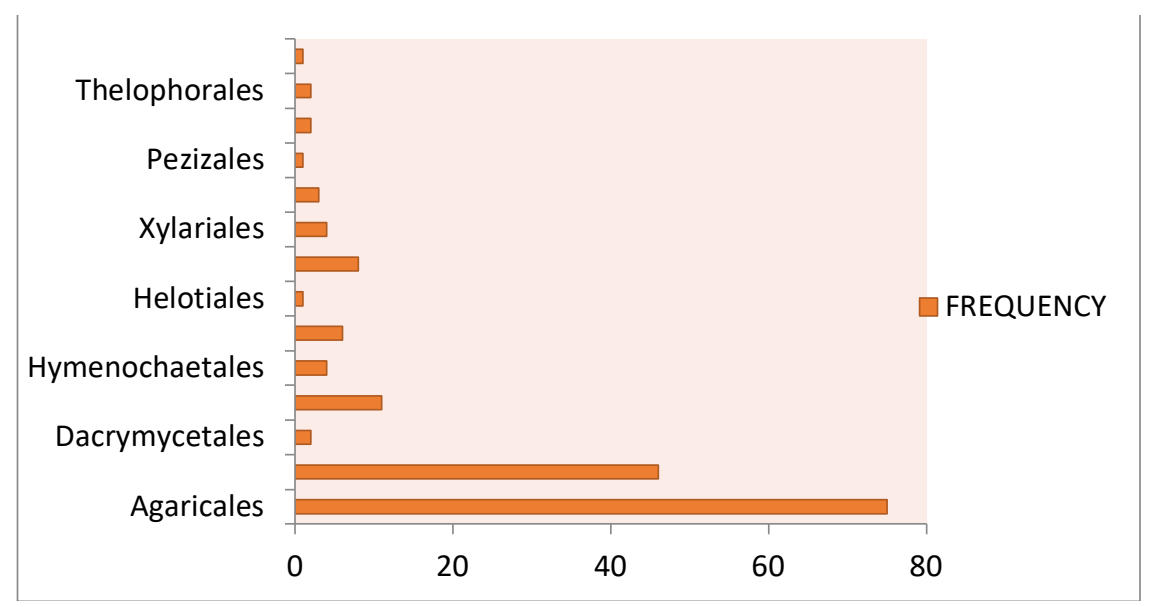

Fig.2: Some Photographs of the collected wild mushroom

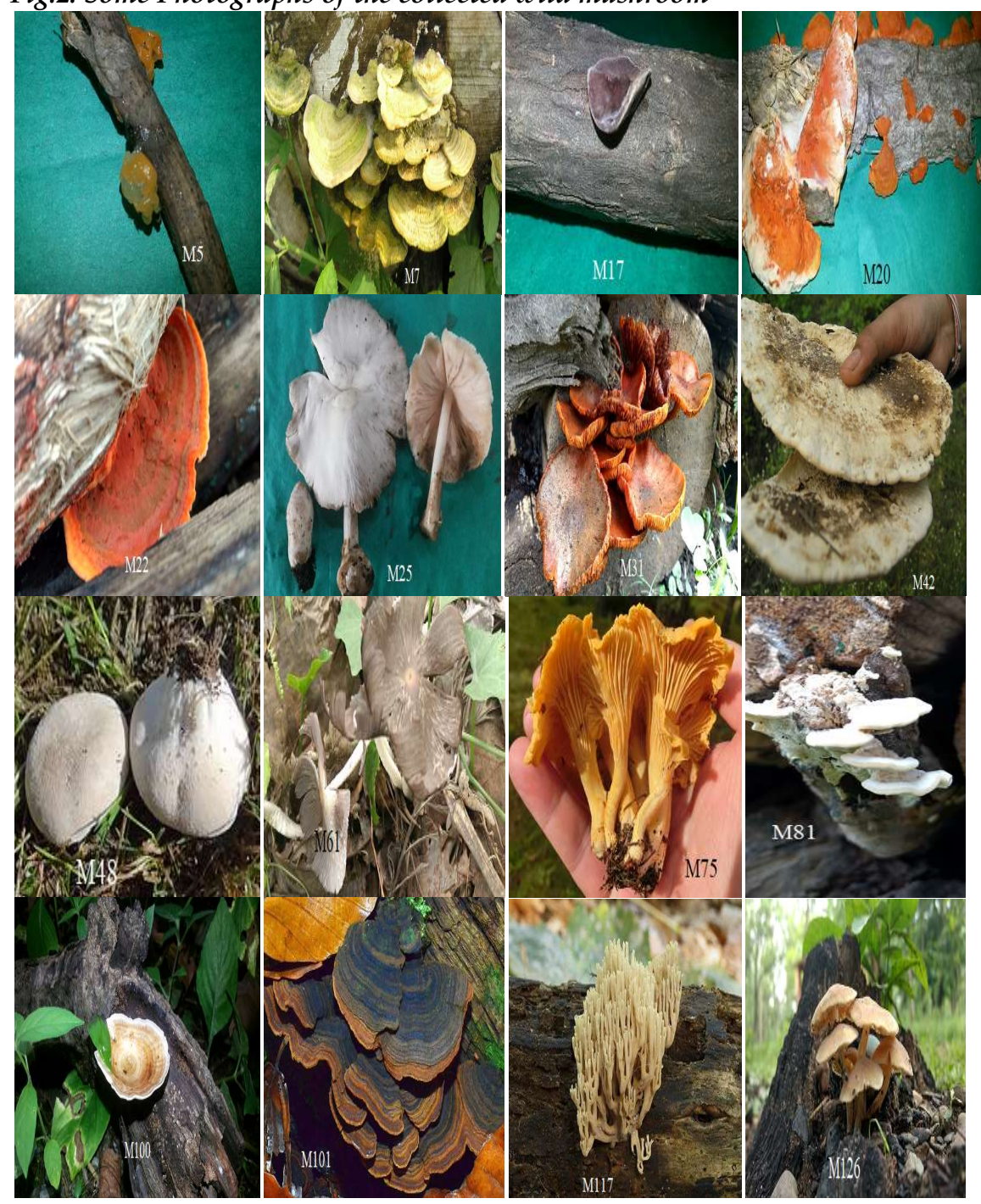



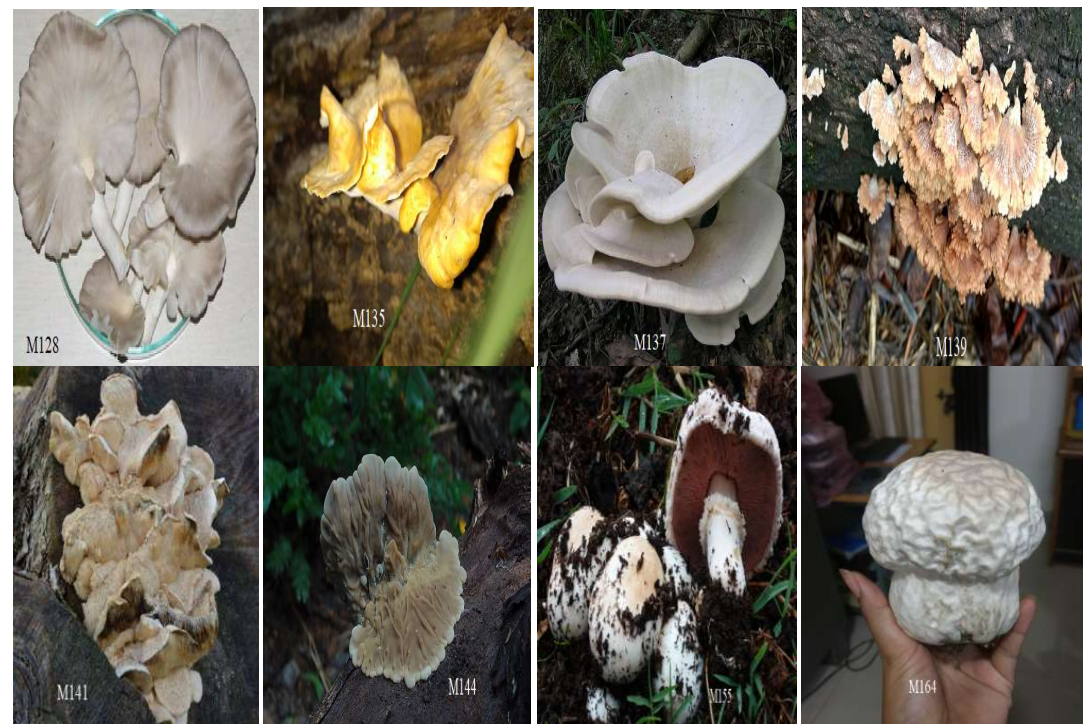

*Scientific names given in Table2.

\section{Conclusions}

This study was aimed to collect and identify wild mushroom that grow naturally in different habitats in Foothill region of Uttarakhand. High diversified mushroom were collected from the foothill where members of Agaricales and Polyporales were maximum indicating that this ecological site contains the high content of organic matter and these two orders has been reported to play important role into the recycling of lingo-cellulosic and hemi-cellulosic organic matter of forest $(13,14)$. Moreover the high rainfall from mid June to July contributes a favorable environment for their growth and activity. During the study it has also being found that majority of the poor population depends on their food on these wild mushrooms. Such a diversified collection has been proved as one of the strongest source of rich nutrient and further study may result in new good sources of antioxidants and pharmaceutical compounds from these wild mushrooms. statement if the study did not report any data.

\section{References}

1. Sharma V. and Jaitly A.K. (2017). Optimization of Growth of Two Wild Species of Pycnoporus Collected from Foothill of Uttarakhand. International Journal of Agriculture Innovations and Research Volume 6(1), pp: 91-94.

2. Bates S.C. (2006). A preliminary checklist of Arizona macrofungi. Canotia 2, pp: 47-78.

3. Atkinson GF. (1961). Studies of American Fungi-Mushrooms, Edible, Poisonous, etc. (2). Hafner Publishing Company, New York.

4. Stametes P. (2000). The Role of Mushroom in Nature, culturing Mushroom Mycelium on Agar Media. In: Growing Gourmet and medicinal mushrooms . Ten Speed Press, Hong Kong.

5. Okoro I.O. et al. (2012) Proximate and mineral analysis of some wild edible mushroom.African Journal of Biotechnology. 11(30), pp. $7720-7724$.

6. Barros L et. al.(2007).;,ICFR Ferreira;, and P Baptista. Food Chemistry.Vol 103:413-419.

7. Thatoi H. and Singdevsachan S.K. (2014). Diversity, nutritional composition and medicinal potential of Indian mushrooms: A review. Vol. 13(4), pp. 523-545.

8. Sarma T.C. et.al.(2010). Wild edible mushrooms used by some ethnic tribes of western Assam. The Bioscan Special Issue 3.pp: 613-625.

9. Natrajan K.C et.al., (2005). Biodiversity of Agarics from Nilgiri Biosphere Reserve, Western Ghats, India. Curr. Sci. 12, $1890-1892$.

10. Christensen C.M. (1972). Common Edible Mushrooms. The University of Minnesota Press. Minneapolis. 
11. Laessoe T. (2000). Mushrooms. Dorling Kindersley Limited London. ISBN 9781405357920

12. Lamaison J.L., Polese J.M. (2005). The Great Encyclopedia of Mushrooms. Konemann Tandem Verlagh Gmbh.

13. Valaskova V., Baldrian P. (2006). Degradation of cellulose and Hemicellulose by the brown rot fungus Piptoporus betulinus. Production of extracellular enzymes and characterization of the major cellulases. Microbiology (Reading):3613-3662.doi:10.1099/mic.0.29149-0. PMID: 17159214.

14. Kumla J. et.al. (2020). Cultivation of Mushroom and their Lignocellulolytic Enzyme Production Through the Utilization of Agro-Industrial Waste. Molecules.25,pp.2811. 